\title{
Dos nuevas citas de Polystichum (Dryopteridaceae) para la FLORA ARgENTINA
}

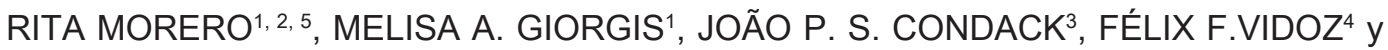 \\ GLORIA E. BARBOZA ${ }^{1,2}$
}

\begin{abstract}
Summary: Two new records of Polystichum (Dryopteridaceae) for the Argentine Flora. In this paper, two new records for the Argentina fern-flora are reported: $P$. subintegerrimum (Hook. \& Arn.) R. Rodr. found in the Patagonian Forests and P. platylepis Fée collected in Misiones; both have been considered endemic for Chile and Brazil, respectively. The species are described and illustrated.
\end{abstract}

\begin{abstract}
Key words: Ferns, endemism, Patagonia, Misiones, Polystichum platylepis, Polystichum subintegerrimum.
Resumen: En este trabajo, dos nuevos taxones de Polystichum se registran para la flora de helechos de Argentina: P. subintegerrimum (Hook. \& Arn.) R. Rodr. hallado en la región de los Bosques Patagónicos y $P$. platylepis Fée colectado en Misiones; hasta ahora, ambas especies eran consideradas endémicas de Chile y Brasil, respectivamente. Se describen e ilustran ambas especies.
\end{abstract}

Palabras clave: Helechos, endemismo, Patagonia, Misiones, Polystichum platylepis, Polystichum subintegerrimum.

Polystichum Roth es uno de los 10 géneros más grandes de helechos del mundo; comprende alrededor de 360-400 spp. de amplia distribución (Zhang, 2012), siendo el este de Asia su centro de origen y diversificación (Li et al., 2004). Sudamérica alberga alrededor de 40 taxones, la mayoría de los cuales ocupan los Andes del Norte (Ecuador, Colombia y Venezuela) y Centrales (Bolivia, Perú y Noroeste de Argentina), constituyendo el principal centro de diversidad en este continente (McHenry \& Barrington, 2014).

1 Instituto Multidisciplinario de Biología Vegetal (UNCCONICET). Casilla de Correo 495, 5000 Córdoba, Argentina.

2 Facultad de Ciencias Químicas, Universidad Nacional de Córdoba. Haya de la Torre y Medina Allende, 5000 Córdoba, Argentina.

3 Museu Nacional, Universidade Federal do Rio de Janeiro. Quinta da Boa Vista, s.n., São Cristóvão, 20940040 Rio de Janeiro, Brasil.

${ }^{4}$ Guardaparque. Parque Nacional Lago Puelo. 9211 Lago Puelo, Chubut, Argentina.

${ }^{5}$ Email: ritamorero@gmail.com
Polystichum es un grupo relativamente homogéneo de helechos que se distingue por el siguiente set de caracteres: rizomas y ejes foliares escamosos; pinnas/pínnulas con la base estrecha, asimétrica, con una aurícula en el borde superior y espínulas en los márgenes de desarrollo variable; soros exindusiados o cubiertos por un indusio redondeado y peltado (Barrington, 1995, 2011). En Argentina, está representado por 8 especies (de la Sota, 1977; de la Sota et al., 1998; Salino \& Ponce, 2008) que exhiben distribución disyunta, tres crecen en el noroeste, sierras pampeanas y sierras de Buenos Aires, y las especies restantes son endémicas de los Bosques Patagónicos e islas del Atlántico sur.

En la actualidad, se está preparando una nueva revisión del género para el sur de Sudamérica. En este contexto, se hallaron en Argentina dos especies $P$. platylepis y $P$. subintegerrimum que no habían sido citadas previamente para nuestro país. El objetivo de este trabajo es aportar descripciones, comentarios e ilustraciones de estas dos nuevas citas. 
Polystichum platylepis Fée, Crypt. Vasc. Brésil 1: 129, t. 41 (40 sic in text), f. 1. 1869. TIPO: [Brasil]. Río de Janeiro, Tijuca, 2-III-1868, Glaziou 2356 (Lectotipo P 00636321! designado por Morero et al. enviado; isolectotipos P 01351317!, P 01351318!; P 00636320!, P 00636322!, P 00636323!, K000590405!). Fig. 1.

Plantas terrestres o saxícolas, medianas a grandes. Rizoma corto, erecto o decumbente, cubierto de escamas; éstas de 5-35 x 1-7 mm, lanceoladas, margen entero, dentado o ciliado, concoloras, castañas a negruzcas o, si bicolores, con una banda central castaño rojizo oscuro y márgenes castaño claro. Frondes laxamente dispuestas, arqueadas, de 30-150 cm long.; pecíolo ca. mitad de la longitud de la fronde, castaño claro en la base y verde oscuro en la porción distal, base densamente cubierta por escamas semejantes a las del rizoma o algo más grandes; lámina 3-4 veces más larga que ancha, lanceolada, 2-pinnada a 2-pinnadapinnatífida, porción distal pinnada acuminada o elongada; raquis verde claro a castaño, escamas de 1-7 x 0,1-0,5 mm, lineares a lanceoladas, margen con dientes curvados, base ciliada y extremo filiforme frecuentemente tortuoso, castaño oscuras a negras y esclerosadas hacia el ápice; pinnas 1040 pares, cortamente pecioluladas, ascendentes, ápice largamente atenuado, siete veces más largas que anchas, las medias de 7-21 x 1,5-5 cm, las basales algo reducidas; pínnulas rectangulares, oblongas, cartáceas, ápice redondeado a obtuso, margen aserrado o crenado, espínulas fuertes en el ápice y la aurícula, ésta notable y aguda, envés con escamas lineares, pequeñas de menos de $2 \mathrm{~mm}$ long.; nerviación libre, nervios 1-3 furcados. Soros sin indusio, ocupando la mayor parte del envés de la lámina. Esporas con perisporio alado, alas estrechas, superficie equinado-perforada (Fig. 3A,B).

Distribución y hábitat: En Argentina, crece en el sotobosque de la selva misionera. En Brasil habita en las zonas montañosas del Sur y Sudeste, en la Sierra da Mantiqueira, Sierra do Mar y Sierra Geral, extendiéndose hacia el Centro-oeste (Estado de Mato Grosso) y Nordeste (sur del Estado de Bahia), donde se lo encuentra próximo a los arroyos en el sotobosque de bosques conservados y en los bordes de los caminos de zonas disturbadas (Condack, 2012, 2015).
Obs. Hasta el momento, esta especie era catalogada como endémica de Brasil (Condack, 2012, Condack, 2015). Al examinar material proveniente de diferentes herbarios se advierte que las determinaciones de estas plantas suelen ser dificultosas y a veces erróneas debido a su similitud con P. montevidense. Ambas especies se diferencian principalmente en el color de las escamas del raquis, siendo castaño claras en $P$. montevidense y oscuras a negruzcas en $P$. platylepis; también por la presencia en la primera de tricomas glandulares en la epidermis foliar, que están ausentes en $P$. platylepis.

Material estudiado. ARGENTINA. Prov. Misiones: Sin otros datos, VII/ 1899, Burmeister 55 (SI 24517). Dpto. Gral. Manuel Belgrano: Ruta Nac. 101, 8 km de Bernardo de Irigoyen hacia San Antonio, Salto Andrecito, 15/X/1996, Morrone et al. 1405 (SI 139443).

Material adicional estudiado. BRASIL. Estado Paraná: Rio Branco do Sul, Itaperuçu, 14-II-1967, Dombrowski 2460 (PACA). Estado Rio Grande Do Sul: Cambará do Sul, Parque Nacional dos Aparados da Serra, 5-VIII-2009, Condack \& Schwartsburd 595 (R, RBR, VT). Estado Santa Catarina: Anita Garibaldi, 13-IV-1963, Reitz \& Klein 14770 (HBR, PACA, RB).

Polystichum subintegerrimum (Hook. et Arn.) R. Rodr., Gayana. Bot. 44: 48. 1987. Aspidium subintegerrimum Hook. \& Arn., Bot. Beechey Voy::52. 1832. Tipo: [Chile]. Concepción, s.f., Hooker \& Arnott s.n. (K 000590468!). Fig. 2.

$\equiv$ Polystichum aculeatum (L.) Schott var. subintegerrimum (Hook. et Arn.) C.Chr., Index Filic.: 576. 1906.

=Polystichum brongniartianum J.Rémy, In Fl. Chil. [Gay] 6: 518. 1854. Tipo: [Chile]. "Chile austral. Concepción”, Gay s.n. No encontrado.

$\equiv$ Aspidium brongniartinum (J.Rémy) Sturm, Abh. Naturhist. Ges. Nürnberg 1: 181.1858.

$\equiv$ Polystichum aculeatum (L.) Schott var. brongniartianum (J.Rémy) C.Chr., Index Filic.: 576. 1906.

Plantas terrestres o saxícolas, medianas. Rizoma erecto cubierto por escamas linear lanceoladas, castaño oscuras. Frondes curvadas, 25-90 (110) x 
R. Morero et al. - Dos nuevas citas de Polystichum para la flora Argentina
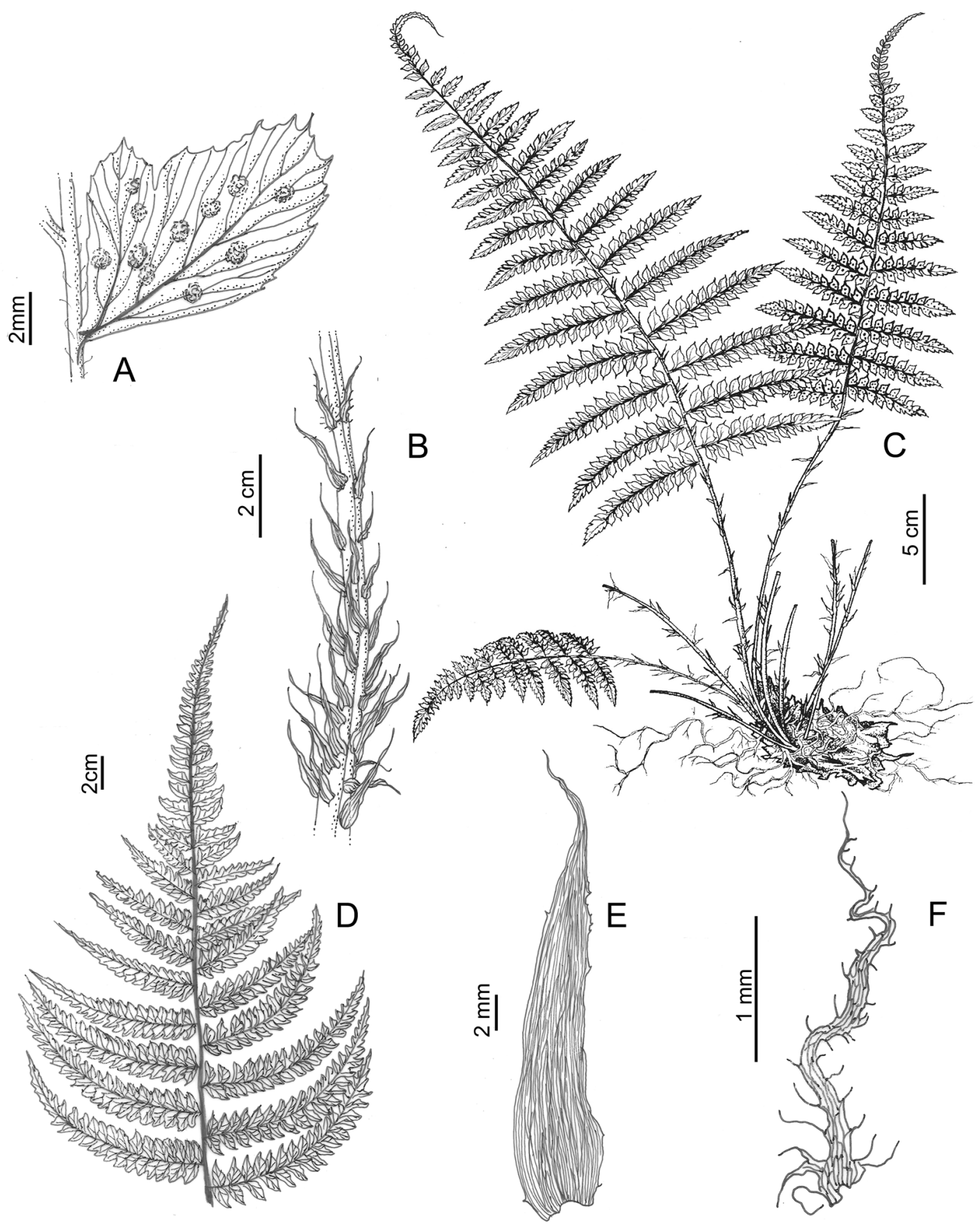

Fig. 1. Polystichum platylepis. A: Detalle de una pínnula mostrando los soros y nerviación. B: Porción basal del pecíolo con escamas. C: Vista general de la planta. D: Porción apical de la lámina. E: Escama de la base del pecíolo. F: Escama del raquis. A, B, D, E, F: Tomado de Condack 2012. C: Dibujó Gonzalo Varela, de Burmeister 55 (SI 24517). 
Bol. Soc. Argent. Bot. 50 (4) 2015

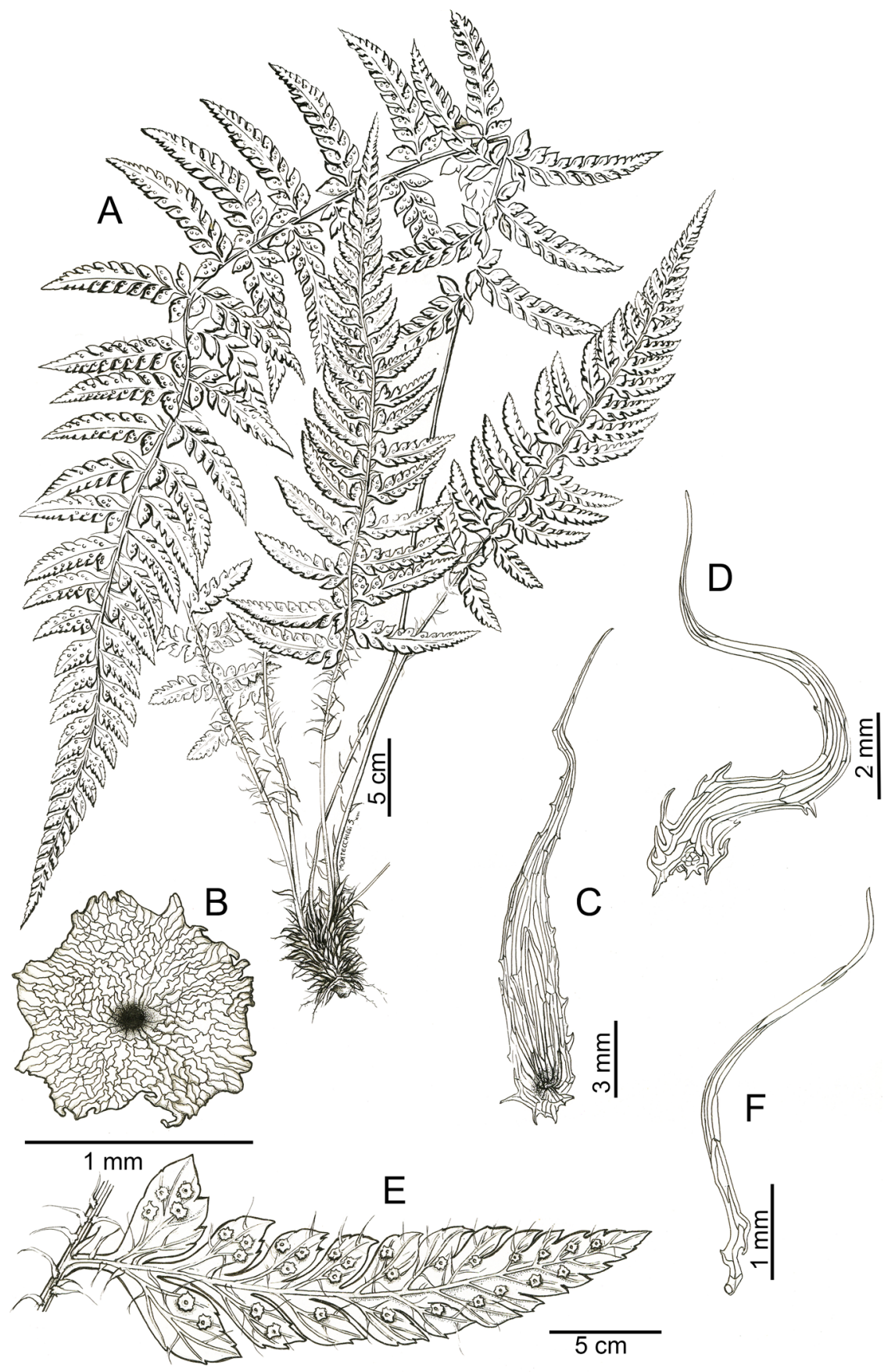

Fig. 2. Polystichum subintegerrimum. A: Aspecto general de la planta. B: Indusio, vista de la cara superior. C: Escama de la región basal del pecíolo. D: Escama del raquis. E: Detalle del envés de una pinna mostrando los soros e indumento. F: Escama del envés de la pinna. Dibujó Silvana Montecchiesi, de Pfister s.n. (CONC 13555). 
7-20 (28) cm. Pecíolo de 1/3 a 1/2 la longitud de la fronde, delgado, con escamas esparcidas y más densas sólo en la base; escamas de 12-20 x 3-5 mm, lanceoladas, margen dentado, concoloras, castaño rojizo, brillantes y bicoloras, centro marrón oscuro a negro y márgenes castaño rojizos. Lámina 4-5 veces más larga que ancha, lanceolada, 2-pinnada, porción apical pinnada, muy elongada; raquis acanalado, con escamas de 2-5 x 0.7-1 mm, triangulares con la base provista de dientes largos, extremo filiforme, concoloras, anaranjado pálido, algunas con el centro esclerosado, negruzco; pinnas tres a cuatro veces más largas que anchas, cartáceas, distanciadas en el raquis y perpendiculares, a veces, ascendentes en la porción apical, las basales algo descendentes e iguales en longitud a las medias o algo reducidas, extremo acuminado (en las plantas jóvenes) a largamente pinnatífido; pínnulas 6-12 (14) pares, frecuentemente el primer par basal más desarrollado, levemente aserradas y con una espínula más desarrollada en el ápice. Soros ocupando más de la mitad del envés de la lámina. Indusio redondeado, margen lacerado. Esporas plano convexas en vista ecuatorial, subcirculares en vista polar; de 58-70 x 48-55 $\mu \mathrm{m}$, perisporio reticulado, superficie fenestrada con espinas simples o ramificadas (Fig. 3C,D).

\section{Distribución y hábitat: Habita en Chile y} Argentina. En el primero, se encuentra desde la provincia de Cauquenes ( $\left.35^{\circ} 59^{\prime} \mathrm{S}\right)$ en el Norte hasta la provincia de Chiloé $\left(42^{\circ} 38^{\prime} \mathrm{S}\right)$ en el Sur y desde el nivel del mar hasta los $1200 \mathrm{~m}$. En nuestro país, vive entre 300-1400 m s.n.m. en la región de los Bosques Patagónicos (Burkart et al, 1999), en Chubut y Río Negro (aunque es muy probable su presencia también en Neuquén), especialmente en los sectores más occidentales (cercanos al límite con Chile), con elevados promedios anuales de precipitación. Se lo encuentra en zonas extremadamente húmedas, en el sotobosque de comunidades boscosas integradas por "coihue" [Nothofagus dombeyi (Mirb.) Oerst.], "palo santo" [Dasyphyllum diacanthoides (Less.) Cabrera], "tineo" (Weinmannia trichosperma Cav.) y "laurel o tepa" [Laureliopsis philippiana (Looser) Schodd], en lugares frecuentemente asociados a ríos, arroyos y cascadas.

Obs. I: Esta especie era considerada endémica para Chile (Rodríguez Ríos, 1995) y fue catalogada como vulnerable por Baeza et al. (1998). Las observaciones a campo y la escasa representación en las colecciones de los herbarios consultados permiten inferir que, en Argentina, presenta baja densidad poblacional y distribución restringida. Cabe agregar, que la ubicación geográfica de las poblaciones encontradas y los sitios de colección de la mayoría de los ejemplares de herbario examinados corresponden a áreas próximas a los pasos andinos de Paso Puelo (Chubut) y Vicente Pérez Rosales (Río Negro); la baja altura de estos pasos cordilleranos los convierte en una fundamental vía de penetración de taxones valdivianos montanos, como es el caso de varias especies de Himenofiláceas (Vidoz et al., 1999; Ponce et al., 2002).

Obs. II: Al analizar la descripción original y el ejemplar tipo de $P$. subintegerrimum observamos que ambos se refieren a plantas grandes (ca. 25 x 1-120 cm long.) que los autores estiman corresponderían a un fenotipo raro y extremo de la especie. Los ejemplares de herbario examinados y las observaciones a campo indican que se trata de poblaciones donde el tamaño de los individuos oscila entre 20-80 cm de long. Corroboran estas observaciones, la información e ilustraciones aportadas por Looser (1968) para esta especie bajo el nombre $P$. brongniartianum.

Material estudiado. ARGENTINA. Prov. Chubut: Dpto. Cushamen, Parque Nacional Lago Puelo, Arroyo de Los Hitos, 10-III-1999, Vidoz 104 (LP). Parque Nacional Lago Puelo, Arroyo del Fuinque, entre Gendarmería y Aguja Norte (Los Hitos), 2-VII-1999, Vidoz 134 (LP). Dpto. Futaleufú, Parque Nacional Los Alerces, sendero entre lago Menéndez y Cisne, 30-X-2008, Biganzoli 1838 (SI). Prov. Río Negro: Dpto. Bariloche, Parque Nacional Nahuel Huapi, Puerto Blest, al costado del arroyo Los Cántaros, 19-III-2008, Morero 244/246 (CORD). Parque Nacional Nahuel Huapi, Puerto Blest, 29-IV-1942, Diem 688 (LP). Laguna Frías al Boquete Pérez Rosales, 19-VI-1940, Pérez Moreau 1010 (BA 34878). Nahuel Huapi, Laguna Frías, 7-II-1934, Burkart 6284 (SI 051217). Parque Nacional Nahuel Huapi, Laguna Frías, VI-1961, Müller \& Reynoso 3580 (BA 82812). Parque Nacional Nahuel Huapi, Laguna Frías, 26-II-1952, Pedersen 1614 (C, MO 170087). 
Bol. Soc. Argent. Bot. 50 (4) 2015
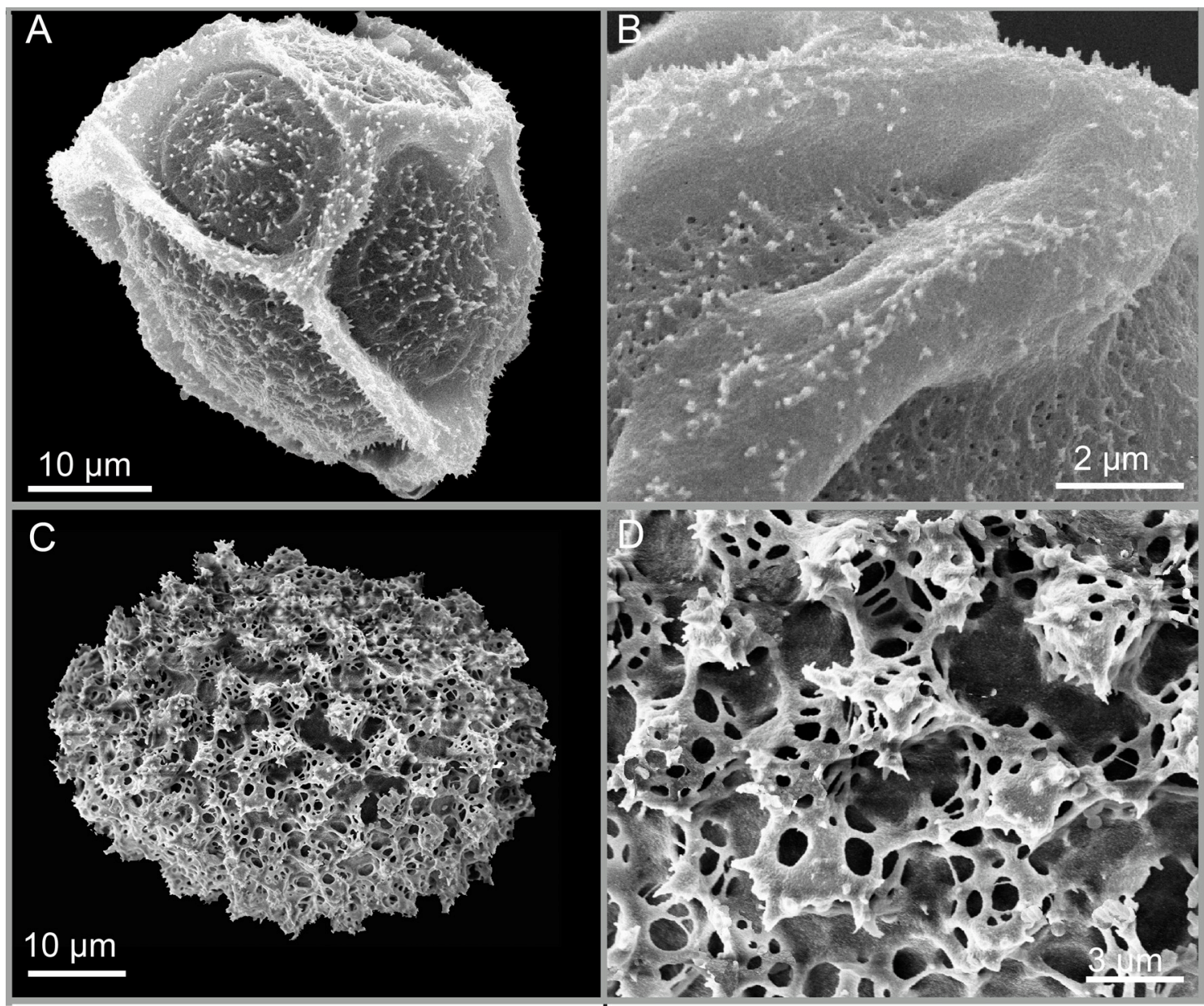

Fig. 3. A, B: Polystichum platylepis, espora en vista distal y detalle del perisporio, respect. [de Condack 652 (R)]. C, D: P. subintegerrimum, espora en vista distal y detalle del perisporio, respectivamente. [de Morero 371 (CORD)].

Material adicional estudiado. CHILE. VII Región del Maule. Prov. Arauco, Comuna Lebu, Isla Mocha. Camino entre el Faro Oeste y La Hacienda, Cordón Central, 14-III-1971. WeldtRodríguez 1105/400 (CONC 35331). VIII Región de Bio Bio. Prov. Concepción: Dpto. Talcahuano, Parque Hualpén, 16-VI-1970. Carrasco 40 (CONC 38005). IX Región de la Araucanía. Prov. Cautín: Comuna de Toltén, Toltén, playa del Pacífico, 4 m s.n.m, 6-III-1942, Hollermayer 1006 (CONC 64117). X Región Los Lagos. Prov. de Chiloé: Comuna de Ancud, 7-VII-1908, Skottsberg 210 (SGO 83470). XI Región de Aysén. Prov. Aysén: Río Blanco, 16-II-2007, García 4039 (CONC 166065). XIV Región de los Ríos. Prov. Ranco: Comuna
Futrono, Llifen (Lago Ranco), 14-VI-1942, Pfister 402 (CONC 4230).

\section{Agradecimientos}

A la Dra. María Mónica Ponce por su desinteresada disposición frente a las consultas y a los revisores anónimos por las valiosas sugerencias. A los Curadores de los Herbarios consultados: BA, BHCB, C, CESJ, CONC, GH, HAS, HBR, HUCS, ICN, K, LP, MBM, MO, NY, P, PACA, R, RB, RBR, SGO, SI, SPF, UPCB, US, VT y especialmente al Herbario del Museo Botánico de Córdoba, por facilitar 


\section{R. Morero et al. - Dos nuevas citas de Polystichum para la flora Argentina}

sus instalaciones, colecciones y bibliografía. A Silvana Montecchiesi y a Gonzalo Varela, un especial agradecimiento por las ilustraciones que acompañan este trabajo.

\section{Biblografía}

BAEZA, M., E. BARRERA, J. FLORES, C. RAMÍREZ \& R. R. RODRÍGUEZ. 1998. Categorías de conservación de Pteridophyta nativas de Chile. Bol. Mus. Nac. Hist. Nat. 47: 23-46.

BARRINGTON, D. S.1995. Polystichum. In: MORAN, R. C. \& R. RIBA. (eds. Pteridofitas), Flora Mesoamericana v.1, pp. 218-225. Universidad Nacional Autónoma de México, Missouri Botanical Garden \& The Natural History Museum (London), México.

BARRINGTON, D. S. 2011. The Fern Genus Polystichum (Dryoperidaceae) in Costa Rica. Ann. Missouri Bot. Gard. 98: 431-446.

BURKAR, T. R., N.O. BÁRBARO, R.O. SÁNCHEZ \& D.A. GÓMEZ. 1999. Ecorregiones de la Argentina. Administración de Parques Nacionales, Buenos Aires.

CONDACK, J. P. S. 2012. Filogenia de Polystichum (Dryopteridaceae) e revisão taxonômica das espécies ocorrentes no Brasil. PhD thesis. Museu Nacional, Universidade Federal do Rio de Janeiro.

CONDACK, J. P. S. 2015. Polystichum in Lista de Espécies da Flora do Brasil. Jardim Botânico do Rio de Janeiro [online]. Disponible en: http:// floradobrasil.jbrj.gov.br/jabot/floradobrasil/ FB90988 [Acceso: 29 Septiembre 2015].

DE LA SOTA, E. R. 1977. Polystichum. In: CABRERA A.L. (ed.), Flora de la provincia de Jujuy, v. 13 (2) pp. 222-227. Colecc. Ci. Inst. Nac. Tecnol. Agropecu., Buenos Aires.

DE LA SOTA, E. R., M. M. PONCE, M. A. MORBELLI \& L.A. CASSÁ DE PAZOS. 1998. Polystichum. In: CORREA M.N. (ed.), Flora Patagónica, v. 4 (1) pp.
356-361. Colecc. Ci. Inst. Nac. Tecnol. Agropecu., Buenos Aires.

LI, C-X., S-G. LU \& Q. YANG. 2004. Asian origin for Polystichum (Dryopteridaceae) based on rbcL sequences. Chin. Sci. Bull. 49: 1146-1150.

LOOSER, G. 1968. Los helechos del género Polystichum Roth en Chile. Notas preliminares. Anales Mus. Hist. Nat. Valparaiso 1: 49-58.

MCHENRY, M. A. \& D. S. BARRINGTON. 2014. Phylogeny and biogeography of exindusiate Andean Polystichum (Dryopteridaceae). Amer. J. Bot. 101: 365-375.

MORERO, R., M. A. MCHENRY, J. P. S. CONDACK \& G. E. BARBOZA. Enviado. Typification and synonymy in Polystichum (Dryopteridaceae) from Chile and Argentina. Acta Bot. Brasil.

PONCE, M. M., K. MEHLTRETER \& E. R. DE LA SOTA. 2002. Análisis biogeográfico de la diversidad pteridofítica en Argentina y Chile continental. Revista Chil. Hist. Nat. 75: 703-717.

RODRIGUEZ R., R. 1995. Pteridophyta. En: Marticorena, C. \& R. R. Rodríguez (eds.), Flora de Chile, Vol. 1, pp. 248-259. Ediciones de la Universidad de Concepción, Concepción.

SALINO, A. \& M.M. PONCE. 2008. Polystichum. In: ZULOAGA, F.O.,

O. MORRONE \& M.J. BELGRANO (eds.), Catálogo de las plantas vasculares del Cono Sur (Argentina, S de Brasil, Chile, Paraguay y Uruguay). Monogr. Syst. Bot. Missouri Bot. Gard. 107: 45-48.

VIDOZ, F. F., L.A.CASSÁ DE PASOS \& E.R. DE LA SOTA. 1999. Paso Puelo, Chubut, Argentina: vía de ingreso de helechos valdivianos. Parodiana 11: 43-48.

ZHANG, L-B. 2012. Taxonomic and nomenclatural notes on the fern genus Polystichum (Dryopteridaceae) in China. Phytotaxa 60: 57-60.

Recibido el 31 de Julio de 2015, aceptado el 19 de setiembre de 2015. 
